\title{
Duality beyond the first loop
}

\author{
Nemanja Kaloper* \\ Department of Physics, University of Waterloo, Waterloo, Ontario, Canada N2L $3 G 1$
}

Krzysztof A. Meissner ${ }^{\dagger}$

CERN, 1211 Geneva 23, Switzerland

(Received 27 May 1997)

\begin{abstract}
In this article we give a calculation of the two-loop $\sigma$-model corrections to the $T$-duality map in string theory. We use the effective action approach, and analyze two-loop corrections in a specific subtraction scheme. Focusing on backgrounds which have a single Abelian isometry, we find the explicit form for the $O\left(\alpha^{\prime}\right)$ modifications of the lowest order duality transformations. Rather surprisingly, the manifest two-loop duality depends crucially on the torsion field. In contrast with the dilaton and metric fields, which are merely passive spectators, the torsion plays a more active role, because of the anomalous couplings to the gauge fields that arise via dimensional reduction. Our results support the interpretation of $T$ duality as an expansion in the inverse string tension $\alpha^{\prime}$, and its order-by-order realization as a manifest symmetry of the full string theory. [S0556-2821(97)06622-8]
\end{abstract}

PACS number(s): 11.25.Hf, 11.25.Db

\section{INTRODUCTION}

Noncompact continuous symmetries different from the trivial diffeomorphism group are very rare in gravitational systems. An example was discovered by Ehlers for the case of the four-dimensional vacuum Einstein gravity with a single Killing vector. These results were later extended by Geroch [1] to the solutions with two Killing isometries, where the symmetry is enhanced to an infinite-dimensional Kac-Moody algebra. In all of these cases, however, noncompact symmetries arise as the hidden symmetries of the equations of motion. The dynamics of gravitational systems admits hidden integrals of motion, which generate the noncompact symmetry by acting on different momentum modes of the gravitational field. In this sense, the Geroch group and its clones are intrinsic to the theory of gravitythey only permute the gravitational degrees of freedom, while leaving the overall theory unaffected.

On the other hand, the gravitational multiplet in string theory has a considerably richer structure than in pure Einstein gravity. In addition to the graviton, it always contains a scalar (dilaton) and an antisymmetric tensor (Kalb-Ramond field, also often referred to as the torsion). Depending on the type of string theory at hand, there may be additional modes, describing the gauge sector of the theory. In this work we will focus on the model-independent sector of the massless modes, leaving the issue of the other modes for later. The low-energy dynamics of these modes is given by an effective action (identical for all consistent string truncations to the model-independent modes in the lowest order), which is covariant, and incorporates the dilaton and torsion effects via nonminimal couplings. The resulting theory can be thought

\footnotetext{
*Electronic address: kaloper@hepvs6.physics.mcgill.ca

†Permanent address: Institute for Theoretical Physics, Hoza 69, 00-689 Warszawa, Poland.

Electronic address: Krzysztof.Meissner@cern.ch
}

of as gravity with corrections, coming from the stringy degrees of freedom. As this interpretation suggests, the symmetries of the Ehlers and Geroch types generalize straightforwardly to the case of the effective actions in string theory [2]. The enhanced complexity of the phase space of string theory, however, may be taken to indicate that string dynamics could be even more symmetric than its pointlike counterpart. Indeed, in string theory there appears yet another class of symmetries. These symmetries have by now become widely known under the general name of duality, and have emerged as an indispensable tool in the quest for a better understanding of string theory (also known as the "theory formerly known as strings"). The impact of these symmetries on the dynamics of string theory is far more profound than the impact of the hidden dynamical symmetries in general relativity, because dualities provide the natural maps between seemingly different string theories, and not only different solutions in the phase space of a single theory. Specifically, the greatest achievement of dualities to date has been the development of the "web of dualities" (see, for example, [3]), which is the basis of the uniqueness proof of string theory, whereby all consistent string constructions have been recognized as the facets of a single fundamental theory, labeled the $M$ theory.

A duality symmetry of interest to us here will be the socalled $T$ duality or string scale-factor duality. At the level of the world-sheet $\sigma$ model, this symmetry has been identified in [4] as a simple Hodge-type duality of a cyclic target coordinate, generated by its flows. The full effect of such transformations on a generic set of target-space degrees of freedom has been exhibited in [5] (in the cases with fewer degrees of freedom, a discrete symmetry of the action was found in [6] and [7]). In these articles it has been shown that when fields depend on only a single coordinate in an arbitrary number of space-time dimensions, the lowest-order Lagrangian exhibits continuous, global $\mathrm{O}(d, d, R)$ symmetry. The symmetry has subsequently been extended to include matter [8] and/or gauge fields $[9,10]$ (we should also mention 
an early analysis in [11], which, however has ignored the dilaton field) and has been identified in all string constructions. There it has also been realized that the continuous $\mathrm{O}(d, d, R)$ symmetry is broken to its discrete $\mathrm{O}(d, d, Z)$ subgroup by instantonlike effects. Finally, it has been discovered that the discrete $\mathbf{Z}_{2}$ subgroup of this group takes a distinguished place in the above-mentioned "web of dualities" [3]. In this context, $T$ duality is not a map in the phase space of a single theory, but instead a map between different theories-an example of the action of such a duality is typeIIA and type-IIB superstring theories compactified on $K 3$ down to six dimensions.

Most of the explicit investigations and applications of $T$ duality were conducted at the one-loop level of the effective approach to string theory, after truncating the action down to the terms of second order in derivatives. Yet it has been indicated, and in some special situations proved, that this symmetry is exact order by order in perturbation theory. In [5] an argument has been given that the symmetry ought to be present to all orders in $\alpha^{\prime}$ in the $\sigma$-model expansion; another argument has been offered in the second reference of [9]. Further, in [7] it has been argued that in the absence of torsion there should be corrections to fields in the next order of $\alpha^{\prime}$ to ensure that the $\beta$ functions vanish, and in $[7,12]$ specific examples to this effect have been given. A more general study of such solutions has been undertaken in [13], where the first explicit proof of $T$ duality to two loops has been given. There, the unique $O\left(\alpha^{\prime}\right)$ effective action consistent with unitarity and $T$ duality invariance has been derived, and the connection between $T$-duality and the "off-shell" $\beta$ function approach has been pointed out (at one loop such a connection was noticed in [14]). Later, other work was done in the attempt to understand the corrections to duality away from conformal points [15].

Last, it has been shown that the lowest-order form of the on-shell $T$-duality map remains unaffected by higher-order $\alpha^{\prime}$ corrections when viewed as a relation between specific conformal field theories (CFT's) [16], some dual solutions in two dimensions [17] and some special supersymmetric solutions [18]. In these cases, the proof relied on special properties of the solutions studied-there either existed an exact, nonperturbative conformal field theory (CFT) formulation, or the solutions were highly symmetric, which protected them from acquiring quantum corrections. A picture which has emerged from these examples is that the $T$-duality map can be expanded as a perturbative series in the inverse string tension $\alpha^{\prime}$.

The overwhelming importance of dualities then seems to beg the question of their validity beyond the first loop. ${ }^{1}$ Since the inclusion of the next-order terms (e.g., curvature squared, etc.) can be very important for the stability of solutions (as

\footnotetext{
${ }^{1}$ We would like to underline here that we consider the loop expansion of the world-sheet $\sigma$ model in the field-theoretic sensehence associating $\alpha^{\prime}$ with the loop counting variable. An alternative interpretation of these corrections, from the target-space viewpoint, is that they are classical, since $\alpha^{\prime}$ measures deviations from the pointlike theory due to the extended structure of strings. We are, however, not going to consider the genus expansion, i.e., the true quantum-theoretic sector of string theory.
}

has been recently pointed out in the case without torsion [19]), and perhaps even elucidate some of the quantummechanical properties of string theory, ${ }^{2}$ it is of interest to test the influence of the $\alpha^{\prime}$ corrections on the $T$-duality maps.

The purpose of this work is to explicitly show that $T$ duality remains manifest [while acquiring $O\left(\alpha^{\prime}\right)$ corrections] even when the two-loop effects are accounted for. The calculation we will present below is a natural extension of Buscher's [4] results beyond the first loop, and is based on identical assumptions: We only restrict our backgrounds to possess a single isometry, compact or not, and take into account all the resulting reduced zero-mass excitations: the metric, dilaton, torsion, and momentum and winding gauge fields. We then take an array of two-loop counterterms, and carry out dimensional reduction on the isometry. The ensuing reduced action contains several terms which appear to violate the duality invariance of the action to $O\left(\alpha^{\prime}\right)$. These terms force us to introduce the $O\left(\alpha^{\prime}\right)$ corrections of the $T$-duality map: Once properly accounted for, $T$ duality reemerges as the invariance of the theory to two loops. A helpful organizing principle, and a consistency check, in this calculation is the gauge invariance of the reduced theory. Inherited from higher dimensions from a subgroup of the full diffeomorphism group and the torsion gauge group, the reduced gauge invariances are anomalous because of the noninvariant split of the components of the torsion potential, which, however, admits a gauge-invariant field strength [10]. The Chern-Simons gauge terms which must be included in the torsion field precisely cancel the gauge dependence of the torsion potential. But they are also crucial for the theory, for without them $T$ duality would have been lost. For example, in heterotic string theory they are partnered up with the vector supermultiplet Yang-Mills Chern-Simons terms, and only in their presence is the generalization of $T$ duality, the full $\mathrm{O}(d, d+n)$ symmetry, involving permutations of the gauge field sector, manifest. Because of this, we can develop a consistency check of our calculations. In the heterotic theory the Yang-Mills Chern-Simons terms arise via a chiral anomaly, which can be computed with the help of the targetspace triangle diagrams. These terms, however, appear only at the one-loop level of the target space theory, and do not receive any further corrections. If $T$ duality is to be valid, similar conditions must be met by the Kaluza-Klein gauge modes as well. Such restrictions on the Kaluza-Klein gauge corrections will turn out to be very helpful in determining the precise form of the $O\left(\alpha^{\prime}\right)$ corrections. We will see that the form of the anomaly can be consistently preserved, and that it does not develop any new, nontrivial, $O\left(\alpha^{\prime}\right)$ corrections. Further, using this we will see how the anomaly transformation rules in fact assist in maintaining $T$ duality to $O\left(\alpha^{\prime}\right)$.

The paper is organized as follows. In the next section we review the one-loop results, in order to set the stage for the

\footnotetext{
${ }^{2} \mathrm{~A}$ good example is the compatibility between duality and anomaly cancellation. To the lowest order, this is illustrated by the explicit appearance of the generalized gauge Chern-Simons terms in the reduced torsion [10], on the one hand dictated by the form of the theory and on the other hand necessitated by duality. While one may expect that this survives quantum corrections, an explicit check is always more convincing.
} 
realm of $O\left(\alpha^{\prime}\right)$ terms. Section III is devoted to the detailed investigation of the $O\left(\alpha^{\prime}\right)$ corrections. Specifically, we will carry out the reduction of the effective action on a single cyclic coordinate, and isolate the terms which break the lowest-order duality symmetry. Using these results, we will derive the explicit form of the $O\left(\alpha^{\prime}\right)$ corrections to the $T$-duality map in Sec. IV. This will in effect prove that $T$ duality is valid to two loops, on any class of backgrounds which admit the lowest-order symmetry. Finally, in the last section we will present our conclusions, and indicate possibilities for future extensions of our results.

\section{ONE-LOOP T DUALITY}

We begin here by reviewing the derivation of the oneloop $T$-duality transformations to set the notation. In doing so, we are following the computation in [10]. The lowestorder term in the effective action of any string theory truncated to only the model-independent zero mass modes is (throughout this paper we use the string frame with $e^{-2 \phi}$ out front, since the symmetry is most simply realized there)

$\Gamma^{(0)}=\int d^{d+1} x \sqrt{\bar{g}} e^{-2 \bar{\phi}}\left\{\bar{R}(\bar{g})+4(\bar{\nabla} \bar{\phi})^{2}-\frac{1}{12} \bar{H}^{2}\right\}$.

Our convention for the signature of the metric is $(-,+, \ldots,+)$, the Riemann curvature is $\bar{R}^{\mu}{ }_{\nu \rho \sigma}$ $=\partial_{\rho} \Gamma^{\mu}{ }_{\nu \sigma}-\cdots$, and the torsion field strength is the antisymmetric derivative of the torsion potential: $\bar{H}_{\mu \nu \rho}$ $=\nabla_{\mu} \bar{B}_{\nu \rho}+$ cyclic. The overbar denotes the quantities in the original, $(d+1)$-dimensional, frame, before we carry out Kaluza-Klein reduction. Note that the definition of the torsion field strength $\bar{H}$ encodes the torsion potential gauge invariance: If we shift the $\bar{B}$ field according to $\bar{B} \rightarrow \bar{B}+d \Lambda$, where $\Lambda$ is an arbitrary one-form, the theory remains unchanged. As a consequence, the Bianchi identity for $\bar{H}$ takes a very simple form: $d \bar{H}=0$.

In the presence of a single Killing isometry, we can carry out the Kaluza-Klein dimensional reduction down to $d$ dimensions. The notion which best charts this dimensional descent is gauge invariance. It is not hard to see that the "offdiagonal" terms in the metric and the torsion potential (those terms mixing the cyclic with non-cyclic indices) must appear as gauge fields in lower dimensions. The reason for this is that there are Abelian gauge invariances associated with them. The translations of the form $y \rightarrow y^{\prime}=y-\omega(x)$ where $y$ is the cyclic coordinate and $x$ 's denote the noncyclic ones, and the special gauge transformations of the two-form $B$ with $\Lambda=\lambda_{y}(x) d y$ leave the theory invariant, but after integrating out the cyclic coordinate $y$, they must appear as the matter sector symmetries, acting on the vectors which emerge from the metric and torsion. Hence the most natural way to carry out the reduction is to ensure that these symmetries are manifest at every step of the calculation-this would guarantee that the final result is symmetric as well. Thus the reduced action will feature two additional gauge fields coupled to the graviton, axion, and dilaton. Moreover, there will also appear an additional scalar field, which is the breathing mode of the cyclic coordinate. In a more general setting with several commuting Killing isometries, there would also appear scalar modes from the the reduction of the two-form torsion potential, residing in the submanifold spanned by the isometries. In our case, they are absent by virtue of their antisymmetry and the fact that we reduce on a single cyclic coordinate.

We can now present the steps of the reduction procedure. As is well known, gauge invariance is enforced as follows. One chooses the reduced metric and dilaton $g_{\mu \nu}$ and $\phi$, respectively, according to

$$
d \bar{s}^{2}=g_{\mu \nu} d x^{\mu} d x^{\nu}+\exp (2 \sigma)\left(d y+V_{\mu} d x^{\mu}\right)^{2} \quad \phi=\bar{\phi}-\frac{1}{2} \sigma .
$$

All the lowering and raising of the indices in the rest of this work will be done with respect to the reduced metric $g_{\mu \nu}$. The vector field $V_{\mu}$ is the standard Kaluza-Klein gauge field, which couples to the momentum modes of the theory. The reduction of the axion field has to be done with more care because of the anomaly which appears in it. Namely, if we look at the naive decomposition of the two-form $\bar{B}=(1 / 2) \bar{B}_{\mu \nu} d x^{\mu} \wedge d x^{\nu}+W_{\mu} d x^{\mu} \wedge d y$ (here $W_{\mu}=\bar{B}_{\mu y}$ is the other gauge field, arising from the "off-diagonal" components of the torsion, and which couples to the winding modes) and attempt to interpret the space-time components $\bar{B}_{\mu \nu}$ as the reduced torsion, we would run into problems with gauge invariance. Whereas they constitute a gauge-invariant tensor with respect to the gauge transformations induced by any $\Lambda=\Lambda_{\mu} d x^{\mu}$ and $\lambda=\lambda_{y} d y$, they are not invariant under the translations along $y$, because of the presence of $d y$ in the decomposition of the original, gauge-invariant, torsion twoform $\bar{B}$. We can see that when $y \rightarrow y^{\prime}=y-\omega(x)$ and $V_{\mu} \rightarrow V_{\mu}^{\prime}+\partial_{\mu} \omega$ (such that the cyclic einbein $E=d y+V_{\mu} d x^{\mu}$ is gauge invariant), the space-time components transform according to $\bar{B}_{\mu \nu} \rightarrow \bar{B}_{\mu \nu}^{\prime}=\bar{B}_{\mu \nu}+W_{\mu} \partial_{\nu} \omega-W_{\nu} \partial_{\mu} \omega$. One may be tempted to alter the reduced torsion potential in analogy with the gauge-invariant reduced metric (2.2). Indeed, it is straightforward to verify that $\hat{B}_{\mu \nu}=\bar{B}_{\mu \nu}-\left(W_{\mu} V_{\nu}-W_{\nu} V_{\mu}\right)$ does not change by the local translations along $y$. However, this field is not invariant under the reduced gauge transformations $\lambda_{y}$. When $W_{\mu} \rightarrow W_{\mu}^{\prime}=W_{\mu}+\partial_{\mu} \lambda_{y}, \quad \hat{B}_{\mu \nu} \rightarrow \hat{B}_{\mu \nu}^{\prime}=$ $\hat{B}_{\mu \nu}-\left(\partial_{\mu} \lambda_{y} V_{\nu}-\partial_{\nu} \lambda_{y} V_{\mu}\right)$. As a result, we see that the two gauge symmetries of the reduced theory are not decoupled, because the reduced torsion potential cannot be simultaneously invariant under both of them. These anomalous transformation properties are at the root of the appearance of the Chern-Simons terms in the reduced theory, as was shown by Maharana and Schwarz [10]. We recall that the quantity which controls the local dynamics of the torsion degrees of freedom is the three-form field strength. It must turn out to be gauge invariant in the reduced theory-because, after all, it will have been derived from the original, higherdimensional theory which is anomaly free. In fact, one can easily define such a gauge-invariant three-form torsion field strength in terms of the reduced quantities, starting with the completely antisymmetric derivative of any of the above two torsion potentials, and then adding the gauge pieces as needed to cancel the anomaly. Further, in order to treat the anomalous transformation properties of the torsion after the dimensional descent in a manifestly symmetric manner, fol- 
lowing $[10]$ one can define the reduced torsion by $B_{\mu \nu}=\bar{B}_{\mu \nu}-(1 / 2)\left(W_{\mu} V_{\nu}-W_{\nu} V_{\mu}\right)$. Then, the gaugeinvariant field strength can be written as $H=d B-(1 / 2) W d V-(1 / 2) V d W$, using the form notation. As we will see below, this approach enforces manifest $T$-duality invariance at the one-loop level, and hence is the correct stepping stone towards extending duality to two loops and beyond. It is then easy to check that the explicit gauge dependence of $H$ precisely cancels the gauge variation of $d B$. As we will see below, the reduced torsion actually appears in the dimensionally reduced action in this form, and hence this field strength is indeed the correct choice for the reduced dynamical variable. To illustrate this, we will now carry out the reduction of Eq. (2.1) in a step-by-step fashion. In that way, we will verify that the three-form torsion field picks up exactly the gauge field couplings prescribed here. Let us now review this, and complete the reduction.

With the ansatz (2.2), one can readily find the tangent space curvature forms. Here we give a detailed account of the results because we will need them later, when we turn our attention to the two-loop corrections. All the quantities are manifestly gauge invariant when we choose to work with the basis forms $e^{a}=e^{a}{ }_{\mu} d x^{\mu}, e^{y}=\exp (\sigma)\left(d y+V_{\mu} d x^{\mu}\right)$, where the vielbein are defined with respect to the reduced metric: $g_{\mu \nu}=\eta_{a b} e^{a}{ }_{\mu} e^{b}{ }_{\nu}$. The vielbein matrix $\left\{e^{a}{ }_{\mu}\right\}$ defines the transformation law between the tangent basis and the holonomic basis $\left\{d x^{\mu}\right\}$. We will also need the unreduced vielbein, denoted by $\bar{e}_{A}^{a}, \bar{e}_{A}^{y}$, and defined by the unreduced metric. Here the capital latin indices run over all the coordinates $\{x\}$ and $y$. It is then easy to verify that $\bar{e}_{\mu}^{a}=e_{\mu}^{a}$, $\bar{e}_{y}^{a}=0, \bar{e}_{\mu}^{y}=\exp (\sigma) V_{\mu}$, and $\bar{e}_{y}^{y}=\exp (\sigma)$. The tangent space curvature components then are

$$
\begin{gathered}
\bar{R}_{a y b y}=\frac{1}{4} e^{2 \sigma} Z_{a b}-\Theta_{a b}, \\
\bar{R}_{a y b c}=-\frac{1}{2} e^{\sigma}\left(\nabla_{a} V_{b c}+2 \nabla_{a} \sigma V_{b c}+2 \nabla_{[c} V_{b] a}\right), \\
\bar{R}_{a b c d}=R_{a b c d}-\frac{1}{4} e^{2 \sigma}\left(\frac{1}{2} V_{a c} V_{b d}-\frac{1}{2} V_{a d} V_{b c}+V_{a b} V_{c d}\right) .
\end{gathered}
$$

The overbar distinguishes between the unreduced and the reduced curvature. We note that after dimensional reduction we can specify a tensor $\mathcal{T}$ by either the tangent space components $T^{a_{1} \cdots a_{p_{1}} \cdots b_{q}}$ or holonomic components $T^{\mu_{1} \cdots \mu_{p}}{ }_{\nu_{1} \cdots \nu_{q}}$, with the vielbein matrix $e^{a}{ }_{\mu}$ defining the change of basis. We introduce the symmetric tensors

$$
\begin{gathered}
Z_{\mu \nu}=V_{\mu \lambda} V_{\nu}^{\lambda}, \quad T_{\mu \nu}=W_{\mu \lambda} W_{\nu}{ }^{\lambda}, \\
\Theta_{\mu \nu}=\exp (-\sigma) \nabla_{\mu} \nabla_{\nu} \exp (\sigma),
\end{gathered}
$$

as a useful shorthand notation. The quantities $Z, T$, and $\Theta$ will be the traces of these tensors. The antisymmetric tensors $V_{\mu \nu}=\partial_{\mu} V_{\nu}-\partial_{\nu} V_{\mu}$ and $W_{\mu \nu}=\partial_{\mu} W_{\nu}-\partial_{\nu} W_{\mu}$ are the standard gauge field strengths of the U(1) gauge fields $V_{\mu}$ and $W_{\mu}$, respectively. The metric-dilaton sector of the action (2.1) is now very easy to obtain. All we need is simply to recall that the Ricci scalar is basis independent, and trace out the tangent space curvature (2.3). The result can be brought in the standard form after substituting the definition of the reduced dilaton in terms of the original one and the modulus $\sigma$, and carrying out a partial integration of some of the terms [10]. The reduction of the three-form kinetic term is slightly subtler because of the anomaly. On the tangent space, $\bar{H}_{\mu \nu \lambda}^{2}=\bar{H}_{A B C}^{T 2}$. Writing out the $y$ indices explicitly, one finds $\bar{H}_{A B C}^{T 2}=\bar{H}_{a b c}^{T} \bar{H}^{T a b c}+3 \bar{H}_{a b y}^{T} \bar{H}^{T a b y}$. But by the decomposition of the two-form torsion, $\bar{H}_{\mu \nu y}=3 \partial_{[\mu} \bar{B}_{\nu y]}=\partial_{\mu} W_{\nu}-$ $\partial_{\nu} W_{\mu}=W_{\mu \nu}$. This yields

$$
\begin{aligned}
\bar{H}_{a b y}^{T} & =\bar{e}_{a}{ }^{A} \bar{e}_{b}{ }^{B} \bar{e}_{y}{ }^{C} \bar{H}_{A B C}=\exp (-\sigma) e_{a}{ }^{\mu} e_{b}{ }^{\nu} \bar{H}_{\mu \nu y} \\
& =\exp (-\sigma) W_{a b} .
\end{aligned}
$$

As a result, $\quad \bar{H}_{a b y}^{T} \bar{H}^{\text {Taby }}=\exp (-2 \sigma) W_{a b} W^{a b}=$ $\exp (-2 \sigma) W_{\mu \nu} W^{\mu \nu}$. Now, the tangent space expression for $\bar{H}_{a b c}^{T}$ is

$$
\begin{aligned}
\bar{H}_{a b c}^{T}= & \bar{e}_{a}{ }^{A} \bar{e}_{b}{ }^{B} \bar{e}_{c}{ }^{C} \bar{H}_{A B C}=e_{a}{ }^{\mu} e_{b}{ }^{\nu} e_{c}{ }^{\lambda} \bar{H}_{\mu \nu \lambda} \\
& +3 e_{[a}{ }^{\mu} e_{b}{ }^{\nu} \bar{e}_{c]}{ }^{y} \bar{H}_{\mu \nu y} \\
= & e_{a}{ }^{\mu} e_{b}{ }^{\nu} e_{c}{ }^{\lambda}\left(\bar{H}_{\mu \nu \lambda}-3 V_{[\lambda} W_{\mu \nu]}\right),
\end{aligned}
$$

where $\bar{H}_{\mu \nu \lambda}=3 \partial_{[\mu} \bar{B}_{\nu \lambda]}=\nabla_{\mu} \bar{B}_{\nu \lambda}+$ cyclic permutations. The pullback of the reduced tangent space three-form $\bar{H}_{a b c}^{T}$ to the holonomic basis is the sought-after gauge-invariant reduced torsion field strength. For note that when we define

$$
H_{\mu \nu \lambda}=\bar{H}_{\mu \nu \lambda}-3 W_{[\mu \nu} V_{\lambda]}
$$

the gauge-dependent pieces in it are invariant under $W_{\mu} \rightarrow W_{\mu}+\partial_{\mu} \lambda_{y}$ but change under the translations along $y$ according to $\delta\left(-3 W_{[\mu \nu} V_{\lambda]}\right)=-3 W_{[\mu \nu} \partial_{\lambda]} \omega$ $=-W_{\mu \nu} \partial_{\lambda} \omega+$ cyclic permutations. This cancels the gauge variation of $3 \partial_{[\mu} \bar{B}_{\nu \lambda]}$, as can be readily verified. Thus $H_{\mu \nu \lambda}$ is precisely the gauge-invariant torsion which we have discussed at the end of the previous paragraph. The gauge anomaly in it takes a slightly unusual guise because it is not symmetric in $V$ and $W$. This, as we have explained above, is because the torsion components $\bar{B}_{\mu \nu}$ are invariant under the reduced Kalb-Ramond U(1) gauge symmetry, and not invariant under the Kaluza-Klein gauge group, generated by the translations along $y$. But as the Bianchi identity for $H_{\mu \nu \lambda}$ yields $\partial_{[\rho} H_{\mu \nu \lambda]} \propto W_{[\rho \mu} V_{\nu \lambda]}$, we can see that the dynamical role of the two gauge fields in the anomaly is equivalent. An egalitarian description of the two symmetries is readily available; we have already introduced it above. All one needs to do is to refer to a different form of the reduced two-form torsion, mentioned before:

$$
B_{\mu \nu}=\bar{B}_{\mu \nu}-\frac{1}{2}\left(W_{\mu} V_{\nu}-W_{\nu} V_{\mu}\right)
$$

In terms of this potential, the gauge-invariant torsion field strength becomes 
$H_{\mu \nu \lambda}=\nabla_{\mu} B_{\nu \lambda}-\frac{1}{2} W_{\mu \nu} V_{\lambda}-\frac{1}{2} V_{\mu \nu} W_{\lambda}+$ cyclic permutations,

which is the component notation for the torsion three-form of the previous paragraph. In this form, the torsion is manifestly $T$-duality invariant, which should occur if duality is to be an exact symmetry of the full quantum theory of strings. Consequently, $H_{A B C}^{2 T}=H_{\mu \nu \lambda} H^{\mu \nu \lambda}+3 \exp (-2 \sigma) T$. This is the last step needed in order to find the reduced action; it is (after dividing $\Gamma_{R}^{(0)}$ by the $y$-volume $\int d y$ )

$$
\begin{aligned}
\Gamma_{R}^{(0)}= & \int d^{d} x \sqrt{g} e^{-2 \phi}\left\{R+4(\nabla \phi)^{2}-(\nabla \sigma)^{2}-\frac{1}{4} e^{2 \sigma} Z\right. \\
& \left.-\frac{1}{4} e^{-2 \sigma} T-\frac{1}{12} H^{2}\right\},
\end{aligned}
$$

with $H$ given in Eq. (2.8). The $T$-duality map is (apart from trivial rescalings) the transformation $\sigma \leftrightarrow-\sigma, V_{\mu} \leftrightarrow W_{\mu}$, and it is obvious that the action (2.10) is invariant under it. The equations of motion which are obtained from varying the action (2.10) (and are simply related to the string $\beta$ functions, in this order) are covariant under $T$ duality: The $\beta$ functions of the dilaton, reduced metric, and torsion are symmetric, the $\beta$ function of the modulus is antisymmetric, and the $\beta$ functions of the gauge fields get interchanged, as expected from the world-sheet $\sigma$-model realization of $T$ duality as a map which exchanges the momentum and winding modes.

An extension of this action for the case of $n$ Abelian isometries, and for general backgrounds which admit nontrivial moduli and gauge fields from both the metric and two-form torsion, takes the following form:

$$
\begin{aligned}
S= & \int d^{d+1-n} x \sqrt{g} e^{-2 \phi}\left\{R+4(\nabla \phi)^{2}+\frac{1}{8} \operatorname{Tr}(\mathcal{L} \nabla \mathcal{M})^{2}\right. \\
& \left.-\frac{1}{4} \mathcal{F}_{\mu \nu}^{T} \mathcal{L} \mathcal{M} \mathcal{L} \mathcal{F}^{\mu \nu}-\frac{1}{12} H_{\mu \nu \lambda}^{2}\right\} .
\end{aligned}
$$

The capital $T$ denotes matrix transposition. The $\sigma$-model fields $\mathcal{M}$ appear after rearranging the scalar moduli fields. The correspondence is given by

$$
\mathcal{M}=\left(\begin{array}{cc}
G^{-1} & -G^{-1} B \\
B G^{-1} & G-B^{T} G^{-1} B
\end{array}\right), \quad \mathcal{L}=\left(\begin{array}{cc}
0 & \mathbf{1} \\
\mathbf{1} & 0
\end{array}\right),
$$

where $G, B$, and $\mathbf{1}$ are $n \times n$ matrices built out of the scalar moduli from the metric and the axion: $G=\left(G_{M N}\right)$ and $B=\left(B_{M N}\right)$. Here the latin indices $\{M, N\}$ run over the internal space. The gauge fields are arranged in the from of the vector multiplet according to

$$
\mathcal{A}_{\mu}=\left(\begin{array}{c}
V^{A}{ }_{\mu} \\
W_{\mu A}
\end{array}\right), \quad \mathcal{F}_{\mu \nu}=\left(\begin{array}{c}
V^{A}{ }_{\mu \nu} \\
W_{\mu \nu A}
\end{array}\right),
$$

and the torsion field strength can be rewritten as

$$
H_{\mu \nu \lambda}=\nabla_{\mu} B_{\nu \lambda}-\frac{1}{2} \mathcal{A}_{\mu}^{T} \mathcal{L} \mathcal{F}_{\nu \lambda}+\text { cyclic permutations. }
$$

Note that $\mathcal{M}^{T}=\mathcal{M}$ and $\mathcal{M}^{-1}=\mathcal{L} \mathcal{M L}$. Thus we see that $\mathcal{M}$ is a symmetric element of $\mathrm{O}(n, n, R)$. Therefore an $\mathrm{O}(n, n, R)$ rotation $\mathcal{M} \rightarrow \Omega \mathcal{M} \Omega^{T}$ and $\mathcal{F} \rightarrow \Omega \mathcal{F}$, while changing $\mathcal{M}$ and $\mathcal{F}$, is a symmetry of the action and the equations of motion. This symmetry is a generalization of the $T$-duality symmetry we have reviewed above $[9,10]$. One must bear in mind, however, that the full $\mathrm{O}(n, n, R)$ group also contains residual gauge degrees of freedom-namely, those diffeomorphisms and gauge transformations of the moduli inherited from the reduction of the metric and torsion. These transformations are the elements of $\mathrm{O}(n, n, R)$ which, viewed in terms of $d \times d$ blocks, are block diagonal and do not mix different tensors but merely rearrange their components. It is easy to see that the net effect is identical to the residual global diffeomorphisms of the internal space. Thus the nontrivial part of the $\mathrm{O}(d, d, R)$ group is the coset $\mathrm{O}(d, d, R) / \mathrm{O}(d, R)$ $\times \mathrm{O}(d, R)$. Quite similarly, in the case of a single isometry which we will study here, the continuous group $\mathrm{O}(1,1, R)$ in fact degenerates down to the two-element group $Z_{2}$ [in this case, we take the group $\mathrm{O}(1,1, R)$ and mod out all the rescalings of the compact coordinate-thus effectively completely determining the value of the modulus by the initial value problem]. Hence, despite its appearance, the only nontrivial $T$-duality symmetry of relevance to our problem is the inversion we have discussed at the end of the previous paragraph.

At this point, we are ready to discuss the two-loop corrections. In the next section, we will present a specific form of the $O\left(\alpha^{\prime}\right)$ terms and, assuming a background with a single isometry, carry out dimensional reduction. The resulting action, as we will see, will contain several terms which will appear to violate $T$ duality, in addition to many terms that will not. Our focus will be on those noninvariant terms, and we will show that they can be reinterpreted as the $O\left(\alpha^{\prime}\right)$ corrections of the lowest-order duality map. Before we go on, we would like to note that to the lowest order, the fields which change under the lowest-order $T$ duality are $\sigma$ (which changes sign), and $V_{\mu}$, and $W_{\mu}$ (which get interchanged). In contrast, the reduced metric $g_{\mu \nu}$, dilaton $\phi$ and torsion $H_{\mu \nu \lambda}$ are invariant-i.e., they are duality singlets. While we would naively expect that they should remain unaffected by duality in higher orders of the $\alpha^{\prime}$ expansion as well, we should observe that the Chern-Simons gauge terms in the torsion may in fact induce nontrivial transformation corrections to it. We will return to this issue later.

\section{REDUCTION OF THE TWO-LOOP ACTION}

In this section, we will present the two-loop corrections, and perform their dimensional reduction on the isometry. A 
complicating factor in our calculations is the presence of the field redefinition ambiguity. Namely, it is well known that the exact form of the $O\left(\alpha^{\prime}\right)$ corrections is not unique, but rather that it depends on the subtraction scheme adopted in string field theory. This ambiguity arises in the following way. The effective action to any given order in the $\alpha^{\prime}$ expansion can be obtained in two ways. One of them is to reverse engineer the $\beta$ functions of the world-sheet $\sigma$ model to get the action whose extrema correspond to the fixed points of the $\sigma$ model. However, the $\beta$ functions are not unique beyond the lowest order, since the renormalization scheme influences the form of the $\alpha^{\prime}$ corrections. The renormalization scheme dependence of the $\beta$ functions is seen as some field redefinition of the effective action, meaning that configurations related by this field redefinition are in fact physically identical. The other way to obtain the effective action is to calculate relevant string amplitudes and expand them to the required order in $\alpha^{\prime}$. In this approach any local field redefinition that respects all the symmetries of the theory and is analytic in $\alpha^{\prime}$ (and is equal to identity in the limit $\alpha^{\prime}=0$ ) yields the same on-shell amplitude, and so the ambiguity in the effective action is in this case even greater than in the $\beta$-function approach. "On shell,', i.e., when both the $\beta$ functions and the functional derivatives of the action vanish, the two descriptions are believed to coincide. The equivalence conjecture simply states that there exist a renormalization scheme and a field redefinition such that the two sets of solutions (constructed from the requirement of vanishing of the $\beta$ functions and the equations of motion) are identical.

Therefore, the appearance of the $O\left(\alpha^{\prime}\right)$ corrections in the effective action can be changed by finite renormalizations of the string world-sheet couplings [20,21]. In a sense, this blurs the notion of string theory quantities as functions of $\alpha^{\prime}$-instead of a single set of solutions, one ends up with equivalence classes, specified by the field redefinitions. Nevertheless, in order to do any calculations one has to adopt a concrete scheme, thus fixing the form of the counterterms in the effective action and the functional dependence on $\alpha^{\prime}$. While many of the counterterms are affected by field redefinitions, a number of them are fixed by the string scattering amplitudes. One could in principle imagine an analogue of the field-theoretic minimal subtraction scheme by simply retaining only the ambiguity-free terms in the action. The merit of such an approach is that it may lead to calculational simplifications for "on-shell" configurations. However, "off shell," i.e., for arbitrary field configurations, the connection between the $\beta$ functions and the functional derivatives of the effective action is a subtle issue. A relation similar to that "on shell" has been suggested in [22], but its validity is far less obvious (although it holds in the known examples-see [20]). In [23,24] relations of this type were used to get the unique "off-shell" action starting from the known two-loop $\beta$ functions. Specifically, because the $\beta$ functions are generally more constrained, and hence less dependent on the redefinitions of fields than the functional derivatives of the effective action, one can use the redefinitions to change the form of the functional derivatives until they are a local linear combination of the $\beta$ functions. This exausts the field redefinition ambiguities, and specifies the two-loop action uniquely_albeit a nonminimal one. Choosing such a scheme hence is perfectly legitimate, although it may appear less appealing initially. But we should underline that while the equations of motion in this scheme may appear far more cumbersome than in the above described "minimal" scheme, the two-loop effective action is defined so that the $\sigma$-model $\beta$ functions are linear combinations of the functional derivatives of the effective action-without constraining the latter to vanish. Thus starting from this action, one can easily write down the complete set of $\beta$ functionsregardless of whether they are "on shell" or "off shell" (i.e., at or away from the conformal point).

Alternatively, a scheme may be specified by simultaneously requiring manifest unitarity in perturbation theory and linear realization of duality [13]. These two requirements have been shown to also specify the action uniquely, at least on the special class of cosmological backgrounds that they were applied to. Surprisingly, the action obtained in this way turned out to be identical to the nonminimal action we have described above-that is, it was precisely the action encoding the local relationship between the $\beta$ functions and the functional derivatives. It was further observed that when one wants to exhibit $T$ duality of the action one makes field redefinitions until the functional derivatives coincide with the $\beta$ functions. This relationship between the $\beta$ functions and $T$ duality was noted in [13] as a very remarkable fact.

The investigation of [13] was done for backgrounds with all but one cyclic coordinates, which were shown to possess the full $\mathrm{O}(d, d)$ symmetry to two loops. The effects of the momentum and winding modes did not arise there because of the symmetry. One can quickly verify that both gauge fields are identically zero on such backgrounds (modulo diffeomorphisms and torsion gauge transformations and provided that none of the isometry generators are null). Our approach here will be based on the same scheme as described in [13]. Here we will extend the analysis to when the gauge degrees of freedom are nontrivial. The effective action to two loops is (we use the same form of the action as the one derived in [13] since it also exhibits manifest $T$ duality in the present case)

$$
\begin{aligned}
\Gamma= & \int \sqrt{\bar{g}} e^{-2 \bar{\phi}}\left\{\bar{R}(\bar{g})+4(\bar{\nabla} \bar{\phi})^{2}-\frac{1}{12} \bar{H}^{2}+\alpha^{\prime} \lambda_{0}\left[-\bar{R}_{\mathrm{GB}}^{2}+16\left(\bar{R}^{\mu \nu}-\frac{1}{2} \bar{g}^{\mu \nu} \bar{R}\right) \bar{\nabla}_{\mu} \bar{\phi} \bar{\nabla}_{\nu} \bar{\phi}-16 \bar{\nabla} \bar{\phi}(\bar{\nabla} \bar{\phi})^{2}+16(\bar{\nabla} \bar{\phi})^{4}\right.\right. \\
& +\frac{1}{2}\left(\bar{R}_{\mu \nu \lambda \rho} \bar{H}^{\mu \nu \alpha} \bar{H}_{\alpha}^{\lambda \rho}-2 \bar{R}^{\mu \nu} \bar{H}_{\mu \nu}^{2}+\frac{1}{3} \bar{R} \bar{H}^{2}\right)-2\left(\bar{\nabla}^{\mu} \bar{\nabla}^{\nu} \bar{\phi} \bar{H}_{\mu \nu}^{2}-\frac{1}{3} \bar{\nabla}^{2} \bar{\phi} \bar{H}^{2}\right)-\frac{2}{3} \bar{H}^{2}(\bar{\nabla} \bar{\phi})^{2}-\frac{1}{24} \bar{H}_{\mu \nu \lambda} \bar{H}_{\rho \alpha} \bar{H}^{\rho \sigma \lambda} \bar{H}_{\sigma}{ }^{\mu \alpha} \\
& \left.\left.+\frac{1}{8} \bar{H}_{\mu \nu}^{2} \bar{H}^{2 \mu \nu}-\frac{1}{144}\left(\bar{H}^{2}\right)^{2}\right]\right\}
\end{aligned}
$$


where we introduce more useful shorthand notation

$$
\bar{H}_{\mu \nu}^{2}=\bar{H}_{\mu \alpha \beta} \bar{H}_{\nu}{ }^{\alpha \beta} \quad \text { and } \quad \bar{H}^{2}=\bar{H}_{\mu \alpha \beta} \bar{H}^{\mu \alpha \beta} .
$$

The action (3.1) is identical to the action obtained by Jack and Jones [24], modulo a boundary term. The parameter $\lambda_{0}$ allows us to move between different string theories: $\lambda_{0}=-1 / 8$ for heterotic, $-1 / 4$ for bosonic, and 0 for superstring. In this sense, our calculations are completely general [although they are, of course, trivial in the case of the superstring-where the $O\left(\alpha^{\prime}\right)$ terms vanish identically and hence the lowest-order $T$ duality does not acquire any corrections; curiously, in this context $T$ duality is not a map between the solutions of a theory but instead a map between different theories]. The $\bar{R}-\bar{H}$ terms include also the Lorentz-Chern-Simons terms which emerge in the heterotic theory to two loops. The curvature-squared terms are arranged in the Gauss-Bonnet combination, $\bar{R}_{\mathrm{GB}}^{2}=\bar{R}_{\mu \nu \lambda \sigma}^{2}-4 \bar{R}_{\mu \nu}^{2}+\bar{R}^{2}$, and hence are manifestly unitary. ${ }^{3}$

While dimensional reduction of this action may appear unsavory at first, it is manageable when carried out on the tangent space. Like in the one-loop case, enforcing reduced gauge symmetries eliminates many of the complicated terms which appear at intermediate steps but cancel in the final answer. Also, we recall that because the action is a scalar, it does not matter in which basis it is written. Therefore, all we need to do is to treat the components of tensors in Eq. (3.1) as tangent space quantities and then decompose them into space-time and internal space subsets. While the calculations are simple, the resulting reduced tensors are defined precisely in terms of the reduced tangent space quantities. The only note of warning is that one has to carefully work out the terms explicitly containing covariant derivatives, reduce them by splitting the connexion, and then pull them back on the tangent space. This in fact is an application of the Young tableaux method for the reduction of irreducible representations of groups-we are reducing $\mathrm{Gl}(d, 1, R)$-invariant quantities to $\mathrm{Gl}(d-1,1, R)$-invariant ones $[$ or in fact $\mathrm{Gl}(d, R)$-invariant terms-for if one views $T$ duality as a solution-generating technique, one may assume $p$-brane-type backgrounds on which the cyclic coordinate is timelike]. After all this has been accomplished, and the cyclic coordinate $y$ integrated out, yielding the reduced action, we can simply replace the reduced tangent space indices with the holonomic ones.

Thus, the Gauss-Bonnet sector of the action yields, after dividing by $l_{y}=\int d y$ and discarding the boundary terms,

$$
\begin{aligned}
\left(1 / l_{y}\right) \bar{R}_{\mathrm{GB}}^{2}= & e^{\sigma}\left\{R_{\mathrm{GB}}^{2}+\frac{3}{16} e^{4 \sigma} Z^{2}-\frac{3}{8} e^{4 \sigma} Z_{\mu \nu}^{2}-4 R \Theta+8 R_{\mu \nu} \Theta^{\mu \nu}+4 e^{2 \sigma} R_{\mu \nu} Z^{\mu \nu}-\frac{1}{2} e^{2 \sigma} R Z-e^{2 \sigma} R_{\mu \nu \lambda \sigma}\left(V^{\mu \nu} V^{\lambda \sigma}+V^{\mu \lambda} V^{\nu \sigma}\right)\right. \\
& +3 e^{2 \sigma} \Theta Z-6 e^{2 \sigma} \Theta_{\mu \nu} Z^{\mu \nu}+e^{-2 \sigma} \nabla_{\mu}\left(e^{2 \sigma} V_{\nu \lambda}\right) \nabla^{\mu}\left(e^{2 \sigma} V^{\nu \lambda}\right)-2 e^{-2 \sigma} \nabla_{\mu}\left(e^{2 \sigma} V^{\mu \nu}\right) \nabla^{\lambda}\left(e^{2 \sigma} V_{\lambda \nu}\right) \\
& \left.+4 V^{\mu \nu} \nabla_{\nu}\left(e^{2 \sigma} V_{\mu \lambda}\right) \nabla^{\lambda} \sigma-4 V^{\mu \nu} \nabla^{\lambda}\left(e^{2 \sigma} V_{\mu \lambda}\right) \nabla_{\nu} \sigma+2 e^{2 \sigma}(\nabla \sigma)^{2} Z-4 e^{2 \sigma} \nabla_{\mu} \sigma \nabla_{\nu} \sigma Z^{\mu \nu}\right\}
\end{aligned}
$$

Next, we notice that the dilaton derivatives are handled with ease. Following the route described in the previous paragraph, we find that the reduction formulas for the derivatives of the dilaton, pulled back to the tangent space, read

$$
\bar{\nabla}_{y} \bar{\nabla}_{y} \bar{\phi}=\nabla_{a} \sigma \nabla^{a} \bar{\phi}, \quad \bar{\nabla}_{a} \bar{\nabla}_{y} \bar{\phi}=-\frac{1}{2} e^{\sigma} V_{a b} \nabla^{b} \bar{\phi}, \quad \nabla_{a} \bar{\nabla}_{b} \bar{\phi}=\nabla_{a} \nabla_{b} \bar{\phi}
$$

Combining these and the expressions (2.3) for the tangent space curvature components, we immediately get the expression for the reduced dilaton-curvature couplings $\bar{R}-\bar{\phi}$ (which are given by $16\left[\bar{R}_{\mu \nu}-(1 / 2) \bar{g}_{\mu \nu} \bar{R}\right] \bar{\nabla}^{\mu} \bar{\phi}^{\nu} \bar{\phi}$ ):

$$
\left(1 / l_{y}\right)(\bar{R}-\bar{\phi})=16\left(R_{\mu \nu}-\frac{1}{2} g_{\mu \nu} R\right) \nabla^{\mu} \bar{\phi} \nabla^{\nu} \bar{\phi}-16\left(\Theta_{\mu \nu}-g_{\mu \nu} \Theta\right) \nabla^{\mu} \bar{\phi} \nabla^{\nu} \bar{\phi}-8\left(Z_{\mu \nu}-\frac{1}{4} g_{\mu \nu} Z\right) \nabla^{\mu} \bar{\phi} \nabla^{\nu} \bar{\phi}
$$

Similarly, the curvature-torsion couplings $(\bar{R}-\bar{H})$ in the original action (given by the expression $\bar{R}-\bar{H}=(1 / 2)$ $\left.\times\left[\bar{R}_{\mu \nu \lambda \sigma} \bar{H}^{\mu \nu \rho} \bar{H}_{\lambda \sigma \rho}-2 \bar{R}_{\mu \nu} \bar{H}^{2 \mu \nu}+(1 / 3) \bar{R} \bar{H}^{2}\right]\right)$ reduce to

\footnotetext{
${ }^{3}$ Manifest unitarity here may be viewed more as another curiosity than a necessity. It is not necessary to require that the effective action (3.1) be manifestly unitary because it represents only an approximation to the complete theory of strings. One could have instead resorted to a different scheme where the curvature corrections, and other terms, do not appear in the unitary form, keeping in mind that in the region of the phase space where unitarity is violated the approximations made to truncate the full theory also break down. This merely indicates that in such limits higher-order corrections must be taken into account. But as we have explained in the text, the action (3.1) also has the property that its functional derivatives are locally related to the $\beta$ functions. It is intriguing to think that unitarity and $T$ duality are equivalent to this reproduction of "off-shell" $\beta$ functions at some deep level.
} 


$$
\begin{aligned}
\left(1 / l_{y}\right)(\bar{R}-\bar{H})= & \frac{1}{2}\left\{R_{\mu \nu \lambda \sigma} H^{\mu \nu \rho} H_{\rho}^{\lambda \sigma}-2 R_{\mu \nu} H^{2 \mu \nu}+\frac{1}{3} R H^{2}+2 \Theta_{\mu \nu} H^{2 \mu \nu}-\frac{2}{3} \Theta H^{2}+e^{-2 \sigma} R_{\mu \nu \lambda \sigma} W^{\mu \nu} W^{\lambda \sigma}-4 e^{-2 \sigma} R_{\mu \nu} T^{\mu \nu}\right. \\
& +e^{-2 \sigma} R T-\frac{1}{2} e^{2 \sigma} H_{\mu \nu \rho} H_{\lambda \sigma}{ }^{\rho}\left(V^{\mu \nu} V^{\lambda \sigma}+V^{\mu \lambda} V^{\nu \sigma}\right)+e^{2 \sigma} H_{\mu \nu}^{2} Z^{\mu \nu}-\frac{1}{12} e^{2 \sigma} H^{2} Z-\frac{3}{4} Z T+3 Z^{\mu \nu} T_{\mu \nu} \\
& -\frac{1}{2} V^{\mu \nu} V^{\lambda \sigma} W_{\mu \lambda} W_{\nu \sigma}-\frac{1}{2} V^{\mu \nu} W_{\mu \nu} V^{\lambda \sigma} W_{\lambda \sigma}+2 \nabla^{\mu} V^{\nu \lambda} W_{\mu}{ }^{\sigma} H_{\nu \lambda \sigma}-2 \nabla_{\lambda} V^{\mu \lambda} W^{\nu \sigma} H_{\mu \nu \sigma}+4 \nabla^{\mu} \sigma V^{\nu \lambda} W_{\mu}{ }^{\sigma} H_{\nu \lambda \sigma} \\
& \left.+4 \nabla^{\lambda} \sigma V^{\nu \mu} W_{\mu}{ }^{\sigma} H_{\nu \lambda \sigma}-6 \nabla_{\lambda} \sigma V^{\mu \lambda} W^{\nu \sigma} H_{\mu \nu \sigma}\right\} .
\end{aligned}
$$

The dilaton-torsion terms $\bar{\phi}-\bar{H}\left(=(2 / 3) \bar{\nabla}^{2} \bar{\phi} \bar{H}^{2}-2 \bar{\nabla}_{\mu} \bar{\nabla}_{\nu} \bar{\phi} \bar{H}^{2 \mu \nu}\right)$ become

$$
\left(1 / l_{y}\right)(\bar{\phi}-\bar{H})=\frac{2}{3} \nabla^{2} \bar{\phi} H^{2}-2 \nabla_{\mu} \nabla_{\nu} \bar{\phi} H^{2 \mu \nu}+\frac{2}{3} \nabla_{\mu} \sigma \nabla^{\mu} \bar{\phi} H^{2}-2 \nabla_{\mu} \bar{\phi} V^{\mu \nu} W^{\lambda \sigma} H_{\nu \lambda \sigma}+2 e^{-2 \sigma} \nabla^{2} T-4 e^{-2 \sigma} \nabla_{\mu} \nabla_{\nu} \bar{\phi} T^{\mu \nu} \text {. }
$$

We recall that to reduce $\left(\bar{H}^{2}\right)^{2}$ we merely need to square the expression for $\bar{H}^{2}$ which we have obtained in the previous section, in the course of deriving the action (2.10). The only remaining terms whose reduction is not trivial are the terms quartic in the torsion $\bar{H}$. Our tangent space formulas for the torsion allow us to write down the following two expressions for these terms:

$$
\left(1 / l_{y}\right) \bar{H}_{\mu \nu \lambda} \bar{H}_{\sigma \rho}^{\nu} \bar{H}^{\sigma \gamma \lambda} \bar{H}_{\gamma}{ }^{\mu \rho}=H_{\mu \nu \lambda} H_{\sigma \rho}^{\nu} H^{\sigma \gamma \lambda} H_{\gamma}{ }^{\mu \rho}+6 e^{-2 \sigma} H_{\mu \nu \rho} H_{\lambda \sigma}{ }^{\rho} W^{\mu \lambda} W^{\nu \sigma}+3 e^{-4 \sigma} T_{\mu \nu} T^{\mu \nu}
$$

and

$$
\left(1 / l_{y}\right) \bar{H}_{\mu \nu}^{2} \bar{H}^{2 \mu \nu}=H_{\mu \nu}^{2} H^{2 \mu \nu}+2 e^{-2 \sigma} H_{\mu \nu \rho} H_{\lambda \sigma}{ }^{\rho} W^{\mu \nu} W^{\lambda \sigma}+4 e^{-2 \sigma} H_{\mu \nu}^{2} T^{\mu \nu}+4 e^{-4 \sigma} T_{\mu \nu} T^{\mu \nu}+e^{-4 \sigma} T^{2} .
$$

With this at hand, we can finally write down the explicit form of the reduced action (2.10). Here we still need to reduce the dilaton, using the formula (2.2). Further, we also need to simplify the terms containing derivatives of the gauge fields. This is accomplished with a number of partial integrations. When we rewrite the action in terms of the shifted dilaton, after some simple algebra, we find the following contributions, which for convenience we present here as sum of several terms:

$$
\Gamma^{(2)}=\Gamma_{1}^{(2)}+\Gamma_{2}^{(2)}+\Gamma_{3}^{(2)}+\Gamma_{4}^{(2)}+\Gamma_{5}^{(2)}
$$

where

$$
\begin{aligned}
\Gamma_{1}^{(2)}= & \int \sqrt{g} e^{-2 \phi}\left\{R+4(\nabla \phi)^{2}-\frac{1}{12} H^{2}+\alpha^{\prime} \lambda_{0}\left[-R_{\mathrm{GB}}^{2}+16\left(R^{\mu \nu}-\frac{1}{2} g^{\mu \nu} R\right) \nabla_{\mu} \phi \nabla_{\nu} \phi-16 \nabla^{2} \phi(\nabla \phi)^{2}+16(\nabla \phi)^{4}\right.\right. \\
& +\frac{1}{2}\left(R_{\mu \nu \lambda \rho} H^{\mu \nu \alpha} H_{\alpha}^{\lambda \rho}-2 R^{\mu \nu} H_{\mu \nu}^{2}+\frac{1}{3} R H^{2}\right)-2\left(\nabla^{\mu} \nabla^{\nu} \phi H_{\mu \nu}^{2}-\frac{1}{3} \nabla^{2} \phi H^{2}\right)-\frac{2}{3} H^{2}(\nabla \phi)^{2}-\frac{1}{24} H_{\mu \nu \lambda} H_{\rho \alpha}^{\nu} H^{\rho \sigma \lambda} H_{\sigma}{ }^{\mu \alpha} \\
& \left.\left.+\frac{1}{8} H_{\mu \nu}^{2} H^{2 \mu \nu}-\frac{1}{144}\left(H^{2}\right)^{2}\right]\right\}
\end{aligned}
$$

which we recognize to have the same form as the original two-loop action (3.1), except that it depends on the reduced fields. The remaining terms describe the gauge- and moduli-dependent corrections. The gauge-dependent terms are as follows:

$$
\begin{aligned}
\Gamma_{2}^{(2)}= & \alpha^{\prime} \lambda_{0} \int d x \sqrt{g} e^{-2 \phi}\left\{-\frac{3}{16} Z^{2} e^{4 \sigma}+\frac{1}{16} T^{2} e^{-4 \sigma}+\frac{3}{8} Z^{\alpha \beta} Z_{\alpha \beta} e^{4 \sigma}+\frac{3}{8} T^{\alpha \beta} T_{\alpha \beta} e^{-4 \sigma}+\frac{3}{2} Z^{\alpha \beta} T_{\alpha \beta}-\frac{3}{8} Z T\right. \\
& \left.-\frac{1}{4}\left(V_{\alpha \gamma} V_{\beta \mu} W^{\alpha \beta} W^{\gamma \mu}+V_{\alpha \beta} W^{\alpha \beta} V_{\gamma \mu} W^{\gamma \mu}\right)\right\}
\end{aligned}
$$

gives the sector quartic in gauge fields, while the gauge-curvature and gauge-torsion couplings are given by 


$$
\begin{aligned}
\Gamma_{3}^{(2)}= & \alpha^{\prime} \lambda_{0} \int d x \sqrt{g} e^{-2 \phi}\left\{\frac{1}{2} R Z e^{2 \sigma}+\frac{1}{2} R T e^{-2 \sigma}+\frac{1}{2} R^{\alpha \beta \mu \nu} V_{\alpha \beta} V_{\mu \nu} e^{2 \sigma}+\frac{1}{2} R^{\alpha \beta \mu \nu} W_{\alpha \beta} W_{\mu \nu} e^{2 \sigma}-2 R^{\alpha \beta} Z_{\alpha \beta} e^{2 \sigma}\right. \\
& -2 R^{\alpha \beta} T_{\alpha \beta} e^{-2 \sigma}+\nabla_{\gamma} V^{\alpha \beta} H_{\alpha \beta \rho} W^{\gamma \rho}+\left(\nabla^{\beta} V_{\beta \alpha}\right) H^{\alpha \rho \gamma} W_{\gamma}^{\rho}-\frac{1}{4} e^{2 \sigma}\left(V_{\alpha \gamma} V_{\beta \mu}+V_{\alpha \beta} V_{\gamma \mu}\right) H^{\alpha \beta \rho} H^{\gamma \mu} \rho \\
& \left.-\frac{1}{4} e^{-2 \sigma}\left(W_{\alpha \gamma} W_{\beta \mu}-W_{\alpha \beta} W_{\gamma \mu}\right) H^{\alpha \beta \rho} H_{\rho}^{\gamma \mu}+\frac{1}{2} e^{2 \sigma} Z^{\mu \nu} H_{\mu \nu}^{2}+\frac{1}{2} e^{-2 \sigma} T^{\mu \nu} H_{\mu \nu}^{2}-\frac{1}{24} e^{2 \sigma} Z H^{2}-\frac{1}{24} e^{-2 \sigma} T H^{2}\right\} .
\end{aligned}
$$

Note that in these expressions there are terms which are not symmetric under the permutation of the gauge fields.

The moduli-dependent self-interactions and the interactions with the dilaton field are

$$
\begin{aligned}
\Gamma_{4}^{(2)}= & \alpha^{\prime} \lambda_{0} \int d x \sqrt{g} e^{-2 \phi}\left\{16\left[\nabla \phi \nabla \sigma+\frac{1}{4}(\nabla \sigma)^{2}\right]\left[2(\nabla \phi)^{2}+\nabla \phi \nabla \sigma+\frac{1}{4}(\nabla \sigma)^{2}\right]+16 \Theta\left(\nabla \phi+\frac{1}{2} \nabla \sigma\right)^{2}\right. \\
& -16 \Theta^{\mu \nu}\left(\nabla_{\mu} \phi+\frac{1}{2} \nabla_{\mu} \sigma\right)\left(\nabla_{\nu} \phi+\frac{1}{2} \nabla_{\nu} \sigma\right)-16\left(\nabla_{\mu} \sigma \nabla^{\mu} \phi+\frac{1}{2}(\nabla \sigma)^{2}+\frac{1}{2} \nabla^{2} \sigma\right)\left(\nabla \phi+\frac{1}{2} \nabla \sigma\right)^{2} \\
& \left.-16 \nabla^{2} \phi\left[\nabla \phi \nabla \sigma+\frac{1}{4}(\nabla \sigma)^{2}\right]\right\},
\end{aligned}
$$

and the interactions of scalars with the curvature, torsion, and gauge fields are

$$
\begin{aligned}
\Gamma_{5}^{(2)}= & \alpha^{\prime} \lambda_{0} \int d x \sqrt{g} e^{-2 \phi}\left\{-4\left(R^{\mu \nu}-\frac{1}{2} g^{\mu \nu} R\right) \nabla_{\mu} \sigma \nabla_{\nu} \sigma+H_{\mu \nu}^{2} \nabla^{\mu} \sigma \nabla^{\nu} \sigma-\frac{1}{6}(\nabla \sigma)^{2} H^{2}+2 \nabla^{\alpha} \phi V_{\rho \alpha} W_{\beta \gamma} H^{\rho \beta \gamma}\right. \\
& +2\left(\nabla_{\gamma} \sigma V_{\alpha \beta}+\nabla_{\beta} \sigma V_{\alpha \gamma}\right) H^{\alpha \beta \rho} W_{\rho}^{\gamma}+2 \nabla^{\beta} \sigma V_{\beta \alpha} W_{\rho \gamma} H^{\alpha \rho \gamma}+2 Z\left(\nabla^{2} \phi+\frac{1}{2} \nabla^{2} \sigma\right) e^{2 \sigma}+2 T\left(\nabla^{2} \phi+\frac{1}{2} \nabla^{2} \sigma\right) e^{-2 \sigma} \\
& -2 Z e^{2 \sigma}\left(\nabla \phi+\frac{1}{2} \nabla \sigma\right)^{2}-2 T e^{-2 \sigma}\left(\nabla \phi+\frac{1}{2} \nabla \sigma\right)^{2}-4 Z^{\alpha \beta} e^{2 \sigma} \nabla_{\alpha}\left(\nabla_{\beta} \phi+\frac{1}{2} \nabla_{\beta} \sigma\right) \\
& \left.-4 T^{\alpha \beta} e^{-2 \sigma} \nabla_{\alpha}\left(\nabla_{\beta} \phi+\frac{1}{2} \nabla_{\beta} \sigma\right)\right\}
\end{aligned}
$$

Note that as we have said above, the contributions to the reduced action which we have listed here contain both terms which are invariant under the one-loop level duality transformations $\sigma \leftrightarrow-\sigma, V_{\mu} \leftrightarrow W_{\mu}$ and terms which are not symmetric under this map. It is these latter terms which we are interested in here. They are the ones forcing us to correct the one-loop duality map with $O\left(\alpha^{\prime}\right)$ contributions. Fortunately, these terms are far fewer than the symmetric ones-hence making our task viable. For completeness sake we will present both classes of terms, before we proceed to discuss the corrections in the next section. In order to separate the two-loop contributions to Eq. (3.10) into one-loop duality-invariant and duality-noninvariant parts, we can apply the transformations, and work out $\Gamma_{\text {inv }}=(1 / 2)\left(\Gamma_{2}+T \Gamma_{2}\right)$ and $\Gamma_{\text {ninv }}=(1 / 2)\left(\Gamma_{2}-T \Gamma_{2}\right)$. Using this, the duality-violating sector of the reduced $O\left(\alpha^{\prime}\right)$ action is

$$
\begin{aligned}
\Gamma_{\text {ninv }}^{(2)}= & \alpha^{\prime} \lambda_{0} \int d x \sqrt{g} e^{-2 \phi}\left\{-4 \nabla_{\mu} \sigma \nabla^{\mu}(\nabla \sigma)^{2}-\nabla_{\mu} \sigma \nabla^{\mu}\left[e^{-2 \sigma} T+e^{2 \sigma} Z\right]+\frac{1}{8}\left[e^{-4 \sigma} T^{2}-e^{4 \sigma} Z^{2}\right]\right. \\
& +\frac{1}{4} H_{\alpha \beta \sigma} H_{\mu \nu}{ }^{\sigma}\left[W^{\alpha \beta} W^{\mu \nu} e^{-2 \sigma}-V^{\alpha \beta} V^{\mu \nu} e^{2 \sigma}\right]+\left[\nabla^{\mu} V^{\nu \lambda} W_{\mu}{ }^{\sigma}-\nabla^{\mu} W^{\nu \lambda} V_{\mu}{ }^{\sigma}\right] H_{\nu \lambda \sigma}+\frac{1}{2}\left[V^{\nu \lambda} W^{\mu \sigma}-W^{\nu \lambda} V^{\mu \sigma}\right] \nabla_{\lambda} H_{\nu \mu \sigma} \\
& \left.+2 \nabla_{\gamma} \sigma V_{\mu \nu} W_{\lambda}^{\gamma} H^{\mu \nu \lambda}+2 \nabla_{\gamma} \sigma W_{\mu \nu} V_{\lambda}^{\gamma} H^{\mu \nu \lambda}\right\}
\end{aligned}
$$

and, finally, the symmetric part of the action is 


$$
\begin{aligned}
\Gamma_{\mathrm{inv}}^{(2)}= & \alpha^{\prime} \lambda_{0} \int d x \sqrt{g} e^{-2 \phi}\left\{-R_{\mathrm{GB}}^{2}+\frac{1}{2}\left[R^{\mu \nu \sigma \rho} H_{\mu \nu \alpha} H_{\sigma \rho}{ }^{\alpha}-2 R^{\mu \nu} H_{\mu \nu}^{2}+\frac{1}{3} R H^{2}\right]+16\left(R^{\mu \nu}-\frac{1}{2} g^{\mu \nu} R\right) \nabla_{\mu} \phi \nabla_{\nu} \phi\right. \\
& -\frac{1}{24} H_{\mu \nu \lambda} H_{\rho \alpha}^{\nu} H^{\rho \sigma \lambda} H_{\sigma}{ }^{\mu \alpha}+\frac{1}{8} H_{\mu \nu}^{2} H^{2 \mu \nu}-\frac{1}{144}\left(H^{2}\right)^{2}-2 \nabla^{\mu} \nabla^{\nu} \phi H_{\mu \nu}^{2}+\frac{2}{3} H^{2} \nabla^{2} \phi-\frac{2}{3} H^{2}(\nabla \phi)^{2}-(\nabla \sigma)^{4}-16(\nabla \sigma \nabla \phi)^{2} \\
& +8(\nabla \sigma \nabla \phi) \nabla^{2} \sigma+4(\nabla \sigma)^{2} \nabla^{2} \phi-4\left(R^{\mu \nu}-\frac{1}{2} g^{\mu \nu} R\right) \nabla_{\mu} \sigma \nabla_{\nu} \sigma+\frac{3}{8}\left[Z^{\alpha \beta} Z_{\alpha \beta} e^{4 \sigma}+T^{\alpha \beta} T_{\alpha \beta} e^{-4 \sigma}\right]-\frac{1}{4} V^{\mu \nu} V^{\lambda \sigma} W_{\mu \lambda} W_{\nu \sigma} \\
& +\frac{1}{2} Z^{\alpha \beta} T_{\alpha \beta}-\frac{1}{8} Z T+\frac{1}{2}\left[R Z e^{2 \sigma}+R T e^{-2 \sigma}\right]-2 R^{\alpha \beta}\left[Z_{\alpha \beta} e^{2 \sigma}+T_{\alpha \beta} e^{-2 \sigma}\right]+\frac{1}{2} R^{\alpha \beta \mu \nu}\left[V_{\alpha \beta} V_{\mu \nu} e^{2 \sigma}+W_{\alpha \beta} W_{\mu \nu} e^{-2 \sigma}\right] \\
& -4 \nabla_{\mu} \nabla_{\nu} \phi\left[Z^{\mu \nu} e^{2 \sigma}+T^{\mu \nu} e^{-2 \sigma}\right]+\left[2 \nabla^{2} \phi-2(\nabla \phi)^{2}-\frac{1}{2}(\nabla \sigma)^{2}\right]\left[Z e^{2 \sigma}+T e^{-2 \sigma}\right]-\frac{1}{24} H^{2}\left[Z e^{2 \sigma}+T e^{-2 \sigma}\right] \\
& \left.+\frac{1}{2} H_{\mu \nu}^{2}\left[Z^{\mu \nu} e^{2 \sigma}+T^{\mu \nu} e^{-2 \sigma}\right]-\frac{1}{4} H_{\alpha \beta \sigma} H_{\mu \nu}{ }^{\sigma}\left[V^{\alpha \beta} V^{\mu \nu} e^{2 \sigma}+W^{\alpha \beta} W^{\mu \nu} e^{-2 \sigma}\right]+2 \nabla_{\gamma} \sigma V_{\mu \nu} W_{\lambda}^{\nu} H^{\mu \gamma \lambda}\right\} .
\end{aligned}
$$

We will not need Eq. (3.17) for the remainder of our calculations. We note, however, that it is an integral part of the subtraction scheme which we have adopted when taking the action (3.1), and it does correspondingly affect the appearance of the solutions to $O\left(\alpha^{\prime}\right)$.

\section{CORRECTIONS TO $T$ DUALITY}

We can finally embark on the study of the corrections (3.16) and their reinterpretation as the modification of the one-loop level $T$-duality map. In order to do so, we will approach the problem from a perturbative standpoint. Namely, the full $O\left(\alpha^{\prime}\right)$ action we have started with is, generically, a good approximation to string dynamics only to order $O\left(\alpha^{\prime}\right)$. This seemingly obvious statement in fact renders valuable information for the understanding of the role of Eq. (3.16). Given the noninvariant terms (3.16) and the oneloop level duality $\sigma \leftrightarrow-\sigma, V_{\mu} \leftrightarrow W_{\mu}$, the natural way to reconcile them is to interpret Eq. (3.16) as the $O\left(\alpha^{\prime}\right)$ terms in the expansion of the exact $T$-duality map, which presumably exists in a complete quantum theory which admits various string theories as its limits. Hence, one ought to be able to incorporate these terms by redefining the duality-invariant one-loop action-in effect shifting the one-loop level fields by amounts proportional to $\alpha^{\prime}$, while preserving any other symmetries the one-loop level theory has. With this in mind, we only need to ensure that the noninvariant terms (3.16) be absorbed to $O\left(\alpha^{\prime}\right)$. Any further deviation away from the perturbative form of $T$ duality, at any higher loop order, that these terms might participate in can be safely ignored from the point of view of the effective action, for we must take into account the influence of other terms beyond the truncation we adopt here, to consistently address such higher-order effects. While our approach may appear limited to the twoloop level, it conforms with the idea that $T$ duality is perturbatively exact. As we will show below, the duality-violating corrections (3.16) will be consistently reincorporated in the one-loop action, respecting all other symmetries, and in particular preserving the form of the one-loop gauge anomaly contributions. At the next order, $\alpha^{\prime 2}$, we expect that the terms violating the two-loop form of duality, which will emerge from our calculations, can be similarly absorbed away as further corrections of the $O\left(\alpha^{\prime 0}\right)$ and $O\left(\alpha^{\prime}\right)$ sectors of the action. The change these terms will induce in the duality map will be quadratic in $\alpha^{\prime}$. Hence such terms can arise as the subleading corrections to the two-loop dualitypreserving contributions in Eq. (3.17) and as $O\left(\alpha^{\prime 2}\right)$ corrections of the one-loop action (2.10). Given the proliferation of the duality-preserving terms, we expect that such analytical cancellations will go on ad infinitum, yielding a Taylor-series expression for the complete $T$-duality map. Note that this does not imply that all the physical variables of string theory must also admit such Taylor-series expansions in $\alpha^{\prime}$. While this may certainly be the case for some of the string degrees of freedom, all we can deduce from the proposed realization of the exact $T$ duality is that the map will not introduce any new $\alpha^{\prime}$ poles in the image of the background it acts on. This means that $T$ duality relates backgrounds it acts on such that both the original and the image admit similar $\alpha^{\prime}$ expansions.

From the results of [13] we expect that the reduced dilaton field $\phi$, a singlet under the one-loop duality, remains inert and does not pick up any $O\left(\alpha^{\prime}\right)$ corrections. This comes about because of the scheme we have adopted for the two-loop terms (3.1). There the reduced dilaton couples to the noninvariant terms exactly the same as to the one-loop terms, implying that the other degrees of freedom must be responsible for restoring the symmetry. This implies that the duality transformation on the dilaton is just a unity, and it remains decoupled from the corrections. However, in a different scheme, there would generally be corrections for the shifted dilaton as well, coming from the fact that the dilaton would interact with the other degrees of freedom via derivative couplings. Moreover, we also expect that the modulus will get corrected by an amount proportional to $(\nabla \sigma)^{2}$, for if we look at the backgrounds with $V_{\mu}=W_{\mu}=H_{\mu \nu \lambda}=0$, our case reduces precisely to that studied in [13].

Now, as we have explained above, we will demonstrate that the noninvariant two-loop contributions will be absorbed as the $O\left(\alpha^{\prime}\right)$ corrections in the one-loop action, yielding a theory manifestly invariant under the corrected $T$-duality transformation. To obtain the precise form of the corrections 
to the duality map, it is instructive to adopt an active approach, whereby we shift all the one-loop level fields by an amount proportional to $\alpha^{\prime}$, and adjust the shifts such that they cancel the noninvariant couplings in Eq. (3.16). This approach will enable us not only to evaluate the corrections, but also to check their consistency with the anomaly cancellation. We will discuss this in more detail below.

We define the shifts of the one-loop degrees of freedom as follows:

$$
\begin{gathered}
\sigma \rightarrow \hat{\sigma}+\alpha^{\prime} \delta \sigma, \quad V_{\mu} \rightarrow \hat{V}_{\mu}+\alpha^{\prime} \delta V_{\mu}, \\
W_{\mu} \rightarrow \hat{W}_{\mu}+\alpha^{\prime} \delta W_{\mu}, \quad H_{\mu \nu \lambda} \rightarrow \hat{H}_{\mu \nu \lambda}+\alpha^{\prime} \delta H_{\mu \nu \lambda} .
\end{gathered}
$$

Starting from the one-loop action (2.10), we can show that the shifts induce the $O\left(\alpha^{\prime}\right)$ correction

$$
\begin{aligned}
\delta \Gamma= & \alpha^{\prime} \int d x \sqrt{g} e^{-2 \phi}\left\{-2 \nabla_{\mu} \sigma \nabla^{\mu}(\delta \sigma)-\frac{e^{2 \sigma}}{2}\right. \\
& \times\left[\delta \sigma V_{\mu \nu}^{2}+V^{\mu \nu} \delta V_{\mu \nu}\right]+\frac{e^{-2 \sigma}}{2}\left[\delta \sigma W_{\mu \nu}^{2}-W^{\mu \nu} \delta W_{\mu \nu}\right] \\
& \left.-\frac{1}{6} H^{\mu \nu \lambda} \delta H_{\mu \nu \lambda}\right\} .
\end{aligned}
$$

In this formula we have replaced the shifted degrees of freedom [denoted by a caret in Eq. (4.1)] by the original oneloop ones, in the spirit of the active interpretation of symmetries. Our aim now is to determine the shifts of the fields such that the correction of the action (4.2) precisely cancels the two-loop noninvariant terms (3.16). Here, however, we should first address the torsion field shift in more detail. As we have mentioned in Sec. II, at the one-loop level the reduced torsion is a duality singlet, just like the reduced dilaton. The dilaton, on the one hand, does not acquire any $O\left(\alpha^{\prime}\right)$ corrections, while even a cursory glance at the twoloop terms in Eq. (3.16) discloses that the torsion field must pick up $O\left(\alpha^{\prime}\right)$ corrections if the $T$ duality is ever to be restored. One may thus wonder just why it may be necessary to correct a field which is only a passive spectator in the arena of $T$ duality, in order to assist the duality itself. This apparent dichotomy has been indicated at the end of the Sec. II. It is resolved when we remember that the reduced torsion couples to the gauge fields via the mixed Chern-Simons term. Given that the vectors transform nontrivially under duality, it is clear that their corrections must play a key role in the restoration of the duality at the two-loop level. Their coupling to the torsion via the Chern-Simons terms, however, also induces the corrections of the torsion field. Thus the torsion assumes the role of a custodian field, with its corrections generated by gauge symmetries and needed to restore duality, while at the same time preserving the form of the anomalous contributions. Indeed, as we will now show, gauge symmetry is very important in determining the form of the torsion corrections.

In Sec. II, we have explained that the components $B_{\mu \nu}$ by themselves do not specify a gauge-invariant tensor. Rather, we must define the reduced torsion potential according to $B_{\mu \nu}=\bar{B}_{\mu \nu}-W_{[\mu} V_{\nu]}$, Eq. (2.8). The reduced field strength $H_{\mu \nu \lambda}=\nabla_{\mu} B_{\nu \lambda}-\frac{1}{2}\left(W_{\mu \nu} V_{\lambda}+V_{\mu \nu} W_{\lambda}\right)+$ cyclic permutations is then fully compliant with the requirement of gauge invariance. The presence of the torsion-gauge-field couplings introduces $O\left(\alpha^{\prime}\right)$ corrections in the torsion, despite the fact that it does not change under the lowest-order $T$ duality. The corrections are necessary because the only way to absorb some of the terms quadratic in the torsion in Eq. (3.16), for example, is to include them in the one-loop torsion kinetic term. Given that the corrections are induced by the torsiongauge-field couplings in the lowest order, they cannot be independent. We have in fact anticipated this, for the following reason. If the corrections to the $H$ field were independent and not merely a consequence of the anomaly combination, we should expect that in higher loop orders the dilaton and the metric also start getting corrected in order to preserve duality-because they couple to the $H$ field, they should also develop independent corrections. On the other hand, if we refer to the world-sheet description of the theory, we can immediately see that the dilaton and curvature do not acquire gauge-field-dependent corrections like the torsion, because they are gauge neutral and anomaly free. Hence they should remain singlets under duality to all orders in perturbation theory, and remain unchanged (modulo string field redefinitions), an apparent contradiction. The only way to resolve this discrepancy is to adopt the above viewpoint and express $\delta H_{\mu \nu \lambda}$ in terms of $\delta V_{\mu}$ and $\delta W_{\mu}$, while maintaining gauge invariance. How are we to accomplish this? First, assuming that $\delta H$ originates only from $\delta V$ and $\delta W$, and imposing that gauge invariance must be respected by the corrections (meaning that the corrections must preserve the functional form of the definition of $\hat{H}$ and the associated Bianchi identity, because the anomaly arises only in the lowest order of the theory and does not receive other nontrivial corrections) we find that, to linear order,

$$
\begin{aligned}
\delta H_{\mu \nu \lambda}= & \nabla_{\mu} \delta B_{\nu \lambda}-\frac{1}{2}\left(W_{\mu \nu} \delta V_{\lambda}+V_{\mu \nu} \delta W_{\lambda}\right)-\frac{1}{2}\left(\delta W_{\mu \nu} V_{\lambda}\right. \\
& \left.+\delta V_{\mu \nu} W_{\lambda}\right) .
\end{aligned}
$$

Since the starting one-loop action is gauge invariant, and the corrections are generated by higher-order terms in perturbation theory, they can only depend on gauge-invariant variables. Therefore, $\delta \sigma, \delta V_{\mu}$, and $\delta W_{\mu}$ must be themselves gauge invariant. However, in Eq. (4.3) we find terms which appear to depend explicitly on the one-loop gauge potentials $V_{\mu}$ and $W_{\mu}$, which are not gauge invariant. Thus, to restore gauge invariance of $\delta H_{\mu \nu \lambda}, \delta B_{\mu \nu}$ must also involve corrections, which will cancel exactly the explicit gauge-dependent terms in $\delta H$. Indeed, we see that there are no such gaugedependent terms anywhere in the two-loop part of the action. So from $B_{\mu \nu}=\bar{B}_{\mu \nu}-W_{[\mu} V_{\nu]}$, we see that

$$
\delta B_{\mu \nu}=\delta \bar{B}_{\mu \nu}-\delta W_{[\mu} V_{\nu]}-W_{[\mu} \delta V_{\nu]} .
$$

If the gauge transformations of the Kaluza-Klein gauge field $V_{\mu} \rightarrow V_{\mu}+\nabla_{\mu} \omega$ are to remain correct in all orders of perturbation theory, which they must for they only correspond to special diffeomorphisms, and no string theory has anomalies which can break these symmetries quantum mechanically, then the gauge transformation rules for all fields must remain the same as they are in the classical limit. The corrections 
must assemble themselves in such a way that the gauge symmetry is preserved beyond the classical limit. If we apply this to the corrected fields $\hat{B}_{\mu \nu}=\bar{B}_{\mu \nu}-\delta \bar{B}_{\mu \nu}$ and $\hat{W}_{\mu}=W_{\mu}$ $-\delta W_{\mu}$, we get

$$
\hat{B}_{\mu \nu}^{\prime}=\hat{B}_{\mu \nu}+\hat{W}_{\mu} \nabla_{\nu} \omega-\hat{W}_{\nu} \nabla_{\mu} \omega
$$

Separating these fields as the classical parts plus the correction, and using the gauge transformation properties of the classical terms, we find that the correction must satisfy an analogous transformation law:

$$
\delta \bar{B}_{\mu \nu}^{\prime}=\delta \bar{B}_{\mu \nu}+\delta W_{\mu} \nabla_{\nu} \omega-\delta W_{\nu} \nabla_{\mu} \omega
$$

Since $\omega$ is just the classical transformation, and the corrections $\delta \bar{B}_{\mu \nu}$ come from the $V$ and $W$ gauge fields, we can see immediately that in order for the last equation to be true we must have

$$
\delta \bar{B}_{\mu \nu}=b_{\mu \nu}+\delta W_{\mu} V_{\nu}-\delta W_{\nu} V_{\mu}
$$

The $\delta W$-dependent corrections are dictated by gauge symmetry, and $b_{\mu \nu}$ is an additional correction to $B$, which must be gauge invariant, and is needed to cancel the terms in Eq. (3.16) that depend on derivatives of $H$. Because we are working with the action to order $\alpha^{\prime}$, by dimensional arguments these terms must be quadratic in derivatives of $V$ and $W$. This determines $b_{\mu \nu}$ up to a constant: $b_{\mu \nu}=c W_{\lambda[\mu} V^{\lambda}{ }_{\nu]}$. Substituting it in the expression for $\delta B$, we find that

$$
\delta B_{\mu \nu}=c W_{\lambda[\mu} V_{\nu]}^{\lambda}+\delta W_{[\mu} V_{\nu]}+\delta V_{[\mu} W_{\nu]} .
$$

Now we can substitute this expression back into the formula for $\delta H$, Eq. (4.3). Rewriting that equation as

$$
\begin{aligned}
\delta H_{\mu \nu \lambda}= & 3 \nabla_{[\mu} \delta B_{\nu \lambda]}-(3 / 2) V_{[\mu \nu} \delta W_{\lambda]} \\
& -(3 / 2) W_{[\mu \nu} \delta V_{\lambda]}-(3 / 2) \delta V_{[\mu \nu} W_{\lambda]} \\
& -(3 / 2) \delta W_{[\mu \nu} V_{\lambda]},
\end{aligned}
$$

after some simple algebra we find that the gauge-dependent terms cancel out:

$$
\delta H_{\mu \nu \lambda}=3 c \nabla_{[\mu}\left(W_{[\nu}^{\rho} V_{\lambda] \rho}\right)-3 V_{[\mu \nu} \delta W_{\lambda]}-3 W_{[\mu \nu} \delta V_{\lambda]} .
$$

Having thus determined $\delta H$, all we need to do now is insert it in the action, and determine $\delta \sigma, \delta V$, and $\delta W$ which cancel Eq. (3.16) and restore $T$ duality.

The unique explicit form of the corrections which lead to the cancellation between Eqs. (3.16) and (4.2) is

$$
\begin{gathered}
\delta \sigma=-2 \lambda_{0}(\nabla \sigma)^{2}-\frac{\lambda_{0}}{4} e^{2 \sigma} Z-\frac{\lambda_{0}}{4} e^{-2 \sigma} T, \\
\delta V_{\alpha}=2 \lambda_{0} V_{\alpha \rho} \nabla^{\rho} \sigma-\frac{\lambda_{0}}{2} H_{\alpha \beta \gamma} W^{\beta \gamma} e^{-2 \sigma}, \\
\delta W_{\alpha}=2 \lambda_{0} W_{\alpha \rho} \nabla^{\rho} \sigma+\frac{\lambda_{0}}{2} H_{\alpha \beta \gamma} V^{\beta \gamma} e^{2 \sigma},
\end{gathered}
$$

with the constant $c=-2 \lambda_{0}$. In order to derive this, all one needs to do is simply collect the like terms in the sum of Eqs. (3.16) and (4.2), demand that they vanish, and find the set of linear equations for the free parameters (which have a nonzero determinant), leading to the solution (4.10). The resulting two terms in the action, the renormalized one-loop part and the invariant two-loop part, are then manifestly invariant under the one-loop level form of the $T$-duality map $\sigma \leftrightarrow-\sigma$, $V_{\mu} \leftrightarrow W_{\mu}$, acting on the corrected fields. If we return then to the original one-loop level degrees of freedom [uncareted ones in Eq. (4.1)], we can interpret the $O\left(\alpha^{\prime}\right)$ shifts as the two-loop corrections to the action of the $T$-duality map on these degrees of freedom. Using this, we can rewrite the two-loop $T$-duality transformation equations as follows:

$$
\begin{aligned}
& \sigma \rightarrow-\sigma-4 \alpha^{\prime} \lambda_{0}(\nabla \sigma)^{2}-\frac{\alpha^{\prime} \lambda_{0}}{2}\left[e^{2 \sigma} Z+e^{-2 \sigma} T\right] \\
& V_{\mu} \rightarrow W_{\mu}-4 \alpha^{\prime} \lambda_{0} W_{\mu \nu} \nabla^{\nu} \sigma-\alpha^{\prime} \lambda_{0} H_{\mu \nu \lambda} V^{\nu \lambda} e^{2 \sigma} \\
& W_{\mu} \rightarrow V_{\mu}-4 \alpha^{\prime} \lambda_{0} V_{\mu \nu} \nabla^{\nu} \sigma+\alpha^{\prime} \lambda_{0} H_{\mu \nu \lambda} W^{\nu \lambda} e^{-2 \sigma}, \\
& H_{\mu \nu \lambda} \rightarrow H_{\mu \nu \lambda}-12 \alpha^{\prime} \lambda_{0} \nabla_{[\mu}\left(W_{\nu}{ }^{\rho} V_{\lambda] \rho}\right) \\
&-12 \alpha^{\prime} \lambda_{0} V_{[\mu \nu} W_{\lambda] \rho} \nabla^{\rho} \sigma-12 \alpha^{\prime} \lambda_{0} W_{[\mu \nu} V_{\lambda] \rho} \nabla^{\rho} \sigma \\
&-3 \alpha^{\prime} \lambda_{0}\left(e^{2 \sigma} V^{\rho \sigma} V_{[\mu \nu}-e^{-2 \sigma} W^{\rho \sigma} W_{[\mu \nu}\right) H_{\lambda] \rho \sigma}
\end{aligned}
$$

Then, the full reduced action, containing all one- and twoloop contributions (2.10), (3.16), and (3.17) is invariant under Eqs. (4.11) to order $\alpha^{\prime}$, as one can check by directly applying these transformation rules. This is our final result.

Before closing this section, we note in passing that the $\beta$-function transformations under duality to two loops can be obtained as an easy by-product of our approach. Since with the help of the corrections (4.1) and (4.10) we can recast the action with the two-loop terms in the form that is invariant under the one-loop duality map, it should be clear that the functional derivatives of this renormalized action transform under duality in the same way as the functional derivatives of the one-loop action. To find explicitly how the functional derivatives transform at the two-loop level, then, all we need to do is to write down the one-loop equations of motion, obtained from varying the action (2.10), and simply replace the original one-loop fields by the corrected fields (4.1). Then, if we expand the corrected fields in $\alpha^{\prime}$, collect the $O\left(\alpha^{\prime}\right)$ contributions, and reverse their overall sign, we get the $O\left(\alpha^{\prime}\right)$ corrections to the transformation rules of the functional derivatives. The next step then is to resort to the local linear relationship between the functional derivatives of Eq. (3.1) with the $\beta$ functions, which we have discussed at the beginning of the Sec. II. Inverting this relationship, and correctly accounting for the transformation properties of this linear map, we would obtain the two-loop form of the transformation properties of the $\beta$ functions under $T$ duality-at and away from the conformal points. Presenting the explicit form of these rules is beyond our goal here, and we defer it to the future. 


\section{CONCLUSION}

In this paper we have examined the $O\left(\alpha^{\prime}\right)$ two-loop corrections in string theory and their effect on, and compliance with, $T$ duality. Our approach was based on the effective field theory of the model-independent zero-mass sector, consisting of the graviton, dilaton, and torsion. The two-loop terms are obtained from the string amplitude calculations, and put in the manifestly unitary form with the string field redefinitions. This has in fact allowed us to ascertain that our calculations apply to different string theories [heterotic, bosonic, and superstring (in which case $T$ duality, of course, is trivially extended to two loops, because the corrections vanish identically)], parametrized by a single constant. Adopting a specific string subtraction scheme and the form of the two-loop effective action this scheme dictates, we have focused on the string backgrounds with a single isometry, and have shown that the theory is invariant under twoloop corrected $T$ duality. We have arrived at the form of the corrections by an iterative reformulation of the $\alpha^{\prime}$ expansion: Any $O\left(\alpha^{\prime}\right)$ terms found to violate the one-loop form of duality were interpreted to induce $O\left(\alpha^{\prime}\right)$ corrections in the original duality map. The explicit form of duality transformations we have found contains terms linear in $\alpha^{\prime}$, which should be thought of as the first subleading terms of the Taylor expansion of the duality map in $\alpha^{\prime}$. Our computations thus are in full agreement with the expectation that $T$ duality is perturbatively exact. One unusual feature of our approach is that solutions of the lowest-order action which have unbroken supersymmetries, and hence are Bogomol'nyi-Prasad-Sommerfield (BPS) states, do not retain their form when the two-loop terms are included. In other words, the two-loop contributions do not cancel among themselves on supersymmetric backgrounds. As a result, when our duality corrections are applied to BPS states, they contain nonvanishing terms to $O\left(\alpha^{\prime}\right)$. While this may sound odd, given the current lore [18], one should remember that while for BPS states there exists a scheme in which the classical solutions are exact to all orders in the $\alpha^{\prime}$ expansion, this, of course, need not be true in any scheme. In fact, those terms among the two-loop corrections which we consider and which do not vanish on BPS backgrounds should be removed by string field redefinitions. As an example, one can take a special class of BPS states, given by the fourdimensional extremal Reissner-Nordström black hole metric and with two purely electric gauge fields $V_{\mu}=W_{\mu}=q / r \delta_{\mu 0}$, and two purely magnetic ones, $v_{\mu}=w_{\mu}=q \cos (\theta) \delta_{\mu 0}$. For this solution, $\phi=\sigma=H_{\mu \nu \lambda}=0$. On the other hand, this solu- tion can be "oxidized" to six dimensions, where the gauge fields $V_{\mu}, v_{\mu}$ are returned to the metric, and $W_{\mu}, w_{\mu}$ to the torsion. Since the field redefinitions to order $\alpha^{\prime}$ depend only on the scalars and rank-2 tensors of the solution which are quadratic in derivatives, with little algebra and the help of the equations of motion, one can verify that the only nontrivial redefinition on this background is $\delta \bar{g}_{\mu \nu}=\alpha^{\prime} \lambda_{0} \kappa \bar{R}_{\mu \nu}$. The parameter $\kappa$ can then be adjusted to remove the correction of the metric component $\bar{g}_{y y}$, or $\sigma$. The remaining terms should then remove the corrections for the metric and gauge fields of the reduced theory. We will not delve on the details here. Suffice it to say that, in some sense, these terms behave like gauge degrees of freedom. Finally, we have indicated in closing how our approach can be utilized to determine transformation properties of the string $\beta$ functions to two loops.

A rather remarkable feature of our calculation has been its reliance on the gauge invariance of the reduced action, where the gauge sector consists of the Kaluza-Klein gauge fields which arise from dimensional reduction of the metric and torsion. The anomalous transformation properties of the torsion, reflected in the Chern-Simons terms in the definition of the torsion field strength, play a crucial role in restoring twoloop duality, while at the same time maintaining gauge invariance and the lowest-order form of the anomaly. This perhaps should not come as a complete surprise. As has been pointed out by Maharana and Schwarz, who discovered the Chern-Simons terms in the reduced theory [10], the anomaly was essential in rendering the one-loop theory $T$ duality invariant. This role of the anomaly seems to persist to two loops, and raises an interesting possibility that the concepts of the anomaly and $T$ duality may somehow be related in the full quantum theory beyond the effective action limit $(M$ theory). An investigation of any such presumed relationship, however, may demand resorting to a different, nonperturbative, approach, due to the complexity of the higher-loop counterterms.

\section{ACKNOWLEDGMENTS}

K.A.M. is grateful to Gabriele Veneziano for earlier collaboration and for discussions on $\mathrm{O}(d, d)$ symmetry. N.K. would like to thank the Theory Division at CERN, where this work has begun, and in particular G. Veneziano for kind hospitality. We are also indebted to R.R. Khuri and R.C. Myers for helpful conversations and comments on the manuscript. This work was supported in part by NSERC of Canada.
[1] R. Geroch, J. Math. Phys. 13, 394 (1972); W. Kinnersley, ibid. 18, 1529 (1977); W. Kinnersley and D. Chitre, ibid. 18, 1538 (1977); 19, 1926 (1978); 19, 2037 (1978).

[2] I. Bakas, Nucl. Phys. B428, 374 (1994).

[3] E. Witten, Nucl. Phys. B443, 85 (1995).

[4] T. Buscher, Phys. Lett. B 194, 59 (1987); 201, 466 (1988).

[5] K. A. Meissner and G. Veneziano, Phys. Lett. B 267, 33 (1991); Mod. Phys. Lett. A 6, 3397 (1991).

[6] G. Veneziano, Phys. Lett. B 265, 287 (1991).
[7] A. A. Tseytlin, Mod. Phys. Lett. A 6, 1721 (1991).

[8] M. Gasperini and G. Veneziano, Phys. Lett. B 277, 256 (1992).

[9] S. F. Hassan and A. Sen, Nucl. Phys. B375, 103 (1992); A. Sen, Phys. Lett. B 271, 295 (1991).

[10] J. Maharana and J. H. Schwarz, Nucl. Phys. B390, 3 (1993).

[11] M. J. Duff, Nucl. Phys. B335, 610 (1990).

[12] J. Panvel, Phys. Lett. B 284, 50 (1992).

[13] K. A. Meissner, Phys. Lett. B 392, 298 (1997). 
[14] P. E. Haagensen, Phys. Lett. B 382, 356 (1996).

[15] J. Balog, P. Forgács, Z. Horváth, and L. Palla, Phys. Lett. B 388, 12 (1996); in Theory of Elementary Particles, Proceedings of the 29th Ahrenshop Symposium, Buckow, Germany, 1995, edited by D. Lust and G. Weigt [Nucl. Phys. B (Proc. Suppl.) 49, 16 (1996)], hep-th/9601091; C. P. Burgess and C. A. Lutken, cond-mat/9611070; P. H. Damgaard and P. E. Haagensen, J. Phys. A 30, 4681 (1997); P. H. Haagensen et al., hep-th/9704157; hep-th/9705105.

[16] E. Kiritsis, Nucl. Phys. B405, 109 (1993); A. Giveon and E. Kiritsis, ibid. B411, 487 (1994).

[17] A. A. Tseytlin, Phys. Lett. B 317, 559 (1993).

[18] R. Kallosh, A. Linde, T. Ortin, A. Peet, and A. Van Proeyen, Phys. Rev. D 46, 5278 (1992); C. Klimcik and A. A. Tseytlin, Phys. Lett. B 323, 305 (1994); R. Kallosh and T. Ortin, Phys. Rev. D 50, 7123 (1994); E. Bergshoeff, I. Entrop, and R. Kallosh, ibid. 49, 6663 (1994); G. T. Horowitz and A. A. Tseytlin, Phys. Rev. Lett. 73, 3351 (1994); Phys. Rev. D 50, 5204
(1994); 51, 2896 (1995); R. Kallosh and A. Linde, ibid. 52, 7137 (1995); M. Cvetič and A. A. Tseytlin, ibid. 53, 5619 (1996); 55, 3907(E) (1997); Phys. Lett. B 366, 95 (1996).

[19] M. Gasperini, M. Maggiore, and G. Veneziano, Nucl. Phys. B494, 315 (1997).

[20] R. R. Metsaev and A. A. Tseytlin, Nucl. Phys. B293, 385 (1987).

[21] D. J. Gross and J. H. Sloan, Nucl. Phys. B291, 41 (1987); C. M. Hull and P. K. Townsend, Phys. Lett. B 191, 115 (1987); D. Zanon, ibid. 191, 354 (1987); I. Jack and D. R. T. Jones, ibid. 193, 449 (1987); K. A. Meissner, J. Pawelczyk, and S. Pokorski, Phys. Rev. D 38, 1144 (1988).

[22] A. B. Zamolodchikov, JETP Lett. 43, 730 (1986); A. M. Polyakov, SLAC Trans-0222, 1986.

[23] N. E. Mavromatos and J. L. Miramontes, Phys. Lett. B 201, 473 (1988).

[24] I. Jack and D. R. T. Jones, Nucl. Phys. B303, 260 (1988). 